\title{
Kommunikative Strategien von Unternehmen zwischen Inszenierung und Adressierung - Eine empirische Analyse eines Imagevideos
}

\author{
Simone Burel/Maria Becker/Laura Kleitsch \\ (Universität Heidelberg)
}

\section{Einleitung}

Unternehmen kommunizieren in verschiedenen symbolischen Interaktionen mit ihren Stakeholdern. Darunter befinden sich, neben Kunden, Shareholdern (Aktionären) oder der Gesellschaft, auch potentielle Mitarbeiter, die geworben bzw. gehalten werden wollen. Dabei muss sich das Unternehmen positiv präsentieren (Inszenierung), um Zuspruch von diesen Anspruchsgruppen zu erhalten. Auf der anderen Seite muss es idealerweise jede einzelne Gruppe in ihrer Lebenswelt kommunikativ ansprechend adressieren (Adressierung), was aufgrund der Vielzahl der möglichen Rezipienten und deren unterschiedlichen Wissenshintergründe (Erfahrungswissen, Fachwissen, Informationsinteresse) zuweilen ein diffiziles Unterfangen ist. Der Artikel beschäftigt sich daher, aufbauend auf der Diskussion dieser theoretischen Ideen, mit der linguistisch-empirischen Analyse eines Imagevideos (BASF Recruiting-Videos) und zeigt die spannungsreiche Kommunikationssituation auf, die sich für das Unternehmen dadurch ergibt.

Die Unternehmenskommunikation bzw. das Kommunikationsmanagement wird inzwischen sorgfältig intern von Unternehmen überwacht und teilweise unter Zuhilfenahme externer Experten (wie Agenturen, Beratungen) optimiert bzw. ausgelagert. Der bewusste Einsatz von Sprache bzw. Kommunikation des Unternehmens ist dahingehend essentiell, da über die sprachliche Gestaltung der ,symbolischen Corporate Persona“ (B.F. Schmid/ B. Lyczek 2008: 98) die Bedeutungen bei Anspruchsgruppen beeinflusst werden können. Natürlich sind die konstituierten Bedeutungen bei den Rezipienten nicht voraussagbar, dennoch kann die Bedeutungsbildung durch spezifische sprachliche (zeichenhafte) Mittel in eine bestimmte Richtung gelenkt werden.

\section{Kommunikationsskripten}

Skripten für kommunikative Standardsituationen widmet sich bereits die Integrierte Kommunikation (vgl. M. Bruhn 2003: 71ff.). B.F. Schmid/ B. Lyczek (2008: 122) beschreiben dagegen ausführlicher mithilfe von E. Goffmans (1969) Ideen, dass sich kommunikative Situationen bis hin zur Mikroebene planen lassen, um kontrollierte Eindrücke zu gestalten: Eine spezifische kommunikative Performance wird dabei vom Unternehmen in einem 
Drehbuch festgelegt; es gibt etwa ein bestimmtes Setting, einen Plot, spezifische Figuren, die in der Kommunikationssituation auftreten, sprachliche, visuelle oder auditive Zeichen, die auf der Ebene der Konnotation einen symbolischen Mehrwert stiften und zur Bedeutungskonstruktion beitragen. Daraus lassen sich Skripten für strategiekonforme „Workable Self Representations“ (B.F. Schmid/ B. Lyczek 2008: 123) des Unternehmens entwickeln. M. Piwinger und H. Ebert (2001: 26) beschreiben des Weiteren eine Reihe von sprachlichen Maßnahmen, die sich im „Werkzeugkasten“ (M. Piwinger/ H. Ebert 2001: 3) des sogenannten Impression Managements finden lassen. Das Impression Management ist eine Art der bestärkenden Kommunikation als „Versuch von Personen oder Institutionen, den Eindruck, den sie auf andere machen, zu steuern und zu kontrollieren. (...) Impression Management ist in diesem Sinne also eine Inszenierungsstrategie“ (M. Piwinger/ H. Ebert 2001: 1). Dabei können mittels einer spezifischen sprachlichen Inszenierung die Inferenzen, die Rezipienten aus dem Text ziehen, im Sinne der gewünschten Bedeutungsbildung beeinflusst werden. M. Piwinger und H. Ebert (2001: 26ff.) fassen folgende Strategien zusammen: Aufbau von Kompetenz, Expertenschaft, Beliebtheit, Status/Prestige, Glaubwürdigkeit. Unter die passiv-defensiven Strategien fällt etwa das Self-Handicapping durch Betonung von Krisen. Assertive Taktiken, die nur kurzfristig von Unternehmen eingesetzt werden können, sind: als Opfer erscheinen; als Vorbild; Idealisierung der eigenen Person; Offenlegung von Schwächen zur Erreichung von Intimität; Angleichungsprozesse (unter Bescheidenheit auftreten); Stereotype bedienen; von überwundenen Schwierigkeiten erzählen; Wahl von Zeichen mit hohem Symbolgehalt (Auto, Globus). Für manche situationalen Darstellungszwänge oder Teilöffentlichkeiten muss das Unternehmen sein sprachliches bzw. kommunikatives Verhalten sogar grundlegend ändern, wenn es diese tatsächlich erreichen will (vgl. M. Piwinger/ H. Ebert 2001: 31). Dies zeigt sich etwa durch die Unternehmensdarstellung bei der Adressierung von potentiellen Auszubildenden im bewusst jugendsprachlich gehaltenen Stil. Darüber hinaus verweisen B.F. Schmid und B. Lyczek (2008: 98) neben der sprachlich-textuellen Repräsentation des Unternehmens auch auf die strategische Eindrucksgestaltung in konkreten Kommunikationssituationen (Kontaktpunkten) oder der Situation selbst, d.h. auf verschiedenen „Bühnen“ wie Pressekonferenzen, Messeauftritten oder Kundengespräche können Auftritte ,inszeniert““ werden. In der Organisationstheorie werden diese Räume etwa als ontische Konstanten angesehen (Rituale, Personen, Raumgestaltung etc.). Deren Konstruktion wird jedoch erst über die Einbeziehung der Metaebene Sprache möglich (vgl. U. Fix 2012: 80), indem gezielte Kommunikationsaktivitäten des Unternehmens geplant, gesteuert und inszeniert werden: Das Unter- 
nehmen antizipiert somit einen idealen Soll-Zustand und bildet diesen durch sprachliche Zeichen in einer Kommunikationssituation als Ist-Zustand ab. Deswegen wird dieser - in Grenzen - idealisiert und negative, polyseme Attributionen, sensible Themen oder offenen Streit vermieden (vgl. E. Goffman 1969: 190ff.). Diese Sprachfolie ist daher eine Art des Imageangebots, denn durch strategisches kommunikatives Design signalisiert das Unternehmen: „so bin ich, und so möchte ich von euch gesehen werden“ (M. Piwinger/ H. Ebert 2001: 5). Durch konkrete Kommunikate werde Information somit übermittelt als „gefrorene“ Kommunikation, die beim „Lesen“ (im Sinne des Auflesens) im Rezipienten wieder sprechend wird. Jede in Informationsmedien kodierte Information kann in einer Reihe möglicher Kommunikationssituationen rezipiert werden (vgl. B.F. Schmid/ B. Lyczek 2008: 120). Der Textproduzent bzw. der Kommunikateur im Unternehmen muss für ein gezieltes Kommunikationsmanagement (mit einer Eindruckssteuerung) jedoch bestimmte Kenntnisse über die sprachgemeinschaftlich geltenden Konventionen in und durch Sprache haben (Präferenzen, Erwartungshaltungen, Rationalität) sowie Kenntnisse über potentielle Rezipienten (deren Handlungssystem, Weltkenntnis und Sprachkenntnis) (vgl. M. Scherner 1997: 51). Er muss ein bestimmtes kognitiv verfügbares Handlungsmuster durch bestimmte kommunikative Mittel aktivieren, indem er spezifische sprachlicher Mittel einsetzt und hofft, durch diese beim Rezipienten eine bestimmte Wirkung zu erreichen (Bewusstseinsveränderung, Kauflust, Bewerbung etc.).

\section{Kommunikations-Vorwissen}

Woher kann der Kommunikateur dieses Kommunikations-Vorwissen nehmen? Unternehmen begegnen diesem Problem häufig mit einem Prozess, den sie unter den Begriff des Wissensmanagements ${ }^{1}$ fassen. Jedoch wird dabei vor allem der gesteuerte Umgang mit bereits vorhandenem Wissen akzentuiert. Der Teilbereich der sprachlichen Wissenskonstituierung dagegen - d.h. die Versprachlichung der fachspezifischen Gegenstände und Sachverhalte (vgl. E. Felder 2008: 270) - wird oft in Theorien des Wissensmanagements vernachlässigt (vgl. I. Nonaka/ H. Takeuchi 1997), ist allerdings von zentraler Bedeutung. ${ }^{2}$

\footnotetext{
${ }^{1}$ „Wissensmanagement kann als geregelter Umgang mit Wissen innerhalb verteilter Systeme von Wissensträgern (Menschen, Dokumente, Dateien) verstanden werden.“ (R. Bromme/ R. Jucks/ R. Rambow 2004: 176)

${ }^{2}$ So plädieren H. Ebert/ K.-P. Konerding (2008: 68) für den Einsatz linguistischer Techniken, z.B. Abstraktionen oder Metaphorisierungen, mit denen zu den impliziten Konstanten eines Unternehmens vorgedrungen wird. Auch mittels narrativer Strategien wird versucht, zu impliziten oder noch nicht versprachlichten Bestandteilen des Wissens hervorzudringen. R. Diaz-Bone (2009: 272) verweist für den Bereich Produktion darauf, dass Unternehmen nicht nur für materielle Instrumentierung (Maschinen) sorgen müssen, sondern auch kognitive Formen (,Wissen“) als immaterielle Instrumentierung berücksichtigen müssen: Dies seien formgebende Strategien (Formatierungen) der Einfassung und Vernetzung der Handlungspraxis (z.B. Erfas-
} 
Denn versucht man, diesem Wissen habhaft zu werden bzw. fragt man nach seinen Konstitutionsbedingungen, kommt man letztlich wieder zur Sprache als dreidimensionale Konstituente für Wissen (vgl. R. Bergmann 2011): Sprache ist das Transfersubjekt des Wissens, Transferobjekt und zugleich auch die Vermittlerin des Wissens. Es verfestigt sich deklarativ durch Kennzahlen, Kapitalformen, Marken oder Produkte. „Personales“ Wissen wird dagegen durch Personen als Wissensträger sprachlich eingebracht, etwa durch Mitarbeiter mit Erfahrungs- und Expertenwissen, Prozess- und Handlungswissen, die wiederum auch externe Repräsentation des personalen Wissens durch Sprache anbringen, z.B. deklarativ in Bedienungsanleitungen oder im Geschäftsbericht. Doch nicht jedes Wissen (gerade prozedurales Wissen wie Prozess- und Handlungswissen von Mitarbeitern) lässt sich extern repräsentieren bzw. nicht jede Repräsentation wird von allen Personen verstanden (vgl. N. Gottschalk-Mazouz 2007: 28f.). Insbesondere das prozedurale Wissen von Mitarbeitern, bei dem viele Teile auch auf impliziten Repräsentationen beruhen (z.B. auch Wahrnehmung von Unternehmenskultur, -werten, -geschichte, atmosphärische Stimmungen, Bilder oder Archetype), lässt sich schwer fassbar machen, benennen sowie in ein gemeinsames, möglichst bereichsunspezifisches Wissen ,„übersetzen“. Beim ganzheitlichen Wissensmanagement in Unternehmen ist daher kommunikationswissenschaftliche bzw. linguistische Expertise gefragt (vgl. W.-A. Liebert 2003: 88) ${ }^{3}$, gepaart mit Fachexpertentum des Unternehmens (vor Ort). Ohne Kenntnis der fachsprachlichen bzw. fachkommunikativen Hintergründe sowie der lebensweltlichen Einordnung der Adressierten kann eine adäquate kommunikative Vermittlung nicht gelingen. Folgendermaßen kann dieses kommunikative Wissen, das für die Eindruckssteuerung und Adressierung in konkreten Kommunikationssituationen essentiell ist, eruiert werden: intern durch Befragungen der Mitarbeiter mittels standardisierten Fragebögen, Einzelinterviews oder Beobachtung von Mitarbeiterverhalten; extern durch Überprüfung von textuellen Maßnahmen (Mitarbeiterzeitung, Stellenanzeigen interne/externe, Repräsentationstexte), Image-Messung mittels Fokusgruppen oder Umfragen (vgl. M. Bruhn 2009: 515ff.). Durch diese gezielte Ansprache von Anspruchsgruppen können Unternehmen ihre Sozialdeixis optimieren, d. h. wie eine persönliche Beziehung zu Adressierten aufgebaut wird. Es geht dabei um das Hineinversetzen in den Adressierten

sung von Arbeitsabläufen, Normierung der zugehörigen Zeiteinheiten, Bewertung von Arbeitsleistung, Spektrum der schriftlichen Fixierungen (Anweisungen), Definition der sprachlichen Formen, grafische Darstellung der Arbeitsabläufe, Einheiten und Leistungsstände.

${ }^{3}$ Liebert (2003) nennt hierbei folgende Schritte: Wissenskonstruktion (Kommunikation und Auslegung), Wissenstransformation (verschiedene Abteilungen interpretieren es unterschiedlich und transformieren Wissen somit; Input wird nicht 1:1 übernommen), Wissensrepräsentation und Wissensdiffusion/Transfer (hierzu zählt auch interne und externe Wissenspräsentation). Idealerweise wird bei diesen Ausführungen davon ausgegangen, dass Sprachverwender dabei in Interaktion stehen und Zeichen dieselben Bedeutungen zuweisen (was als Wissen gilt). 
(Perspektivübernahme), die Verwendung von situational angemessener, direkter Ansprache oder spezifischen Höflichkeitsformen. Anspruchsgruppen können somit ihre Perspektive sowie ihren Erfahrungs-/Erwartungshorizont wiederfinden. ${ }^{4}$ Das Unternehmen weiß um deren Werte, Wünsche, Bedürfnisse, Ansprüche oder Interessen. Relevant ist dieser Perspektivenwechsel vor allem dann, wenn eigentlich divergierende Maximen (Unternehmenssicht vs. Mitarbeitersicht) das Ziel bestimmen.

\section{Empirische Analyse eines Imagevideos der BASF}

Aus den vorangehenden Ausführungen wurde bereits deutlich, dass sich die (externe) Unternehmenskommunikation grundsätzlich zweifach auszurichtet: Zum einen geht es darum, das eigene Unternehmen positiv zu präsentieren, gleichzeitig gilt es aber auch, verschiedene Rezipienten bzw. Rezipientengruppen anzusprechen, denn eine gezielte Ansprache bzw. Adressierung ist ausschlaggebend für deren Zuspruch. Die Verfolgung beider Ansprüche bzw. Zielsetzungen zeigt sich auch in dem Imagevideo der BASF, das im Folgenden unter multimedial-sprachlichen Aspekten analysiert werden soll. Das Video wurde für das Recruiting von Praktikanten im Bereich Personalmarketing und Social Media produziert und im Mai 2013 auf der Videoplattform Youtube veröffentlicht, wo es bisher bereits fast 9000 Mal aufgerufen wurde. Das Video ist 1 Minute und 45 Sekunden lang und wurde für die Analyse zunächst sowohl auditiv als auch visuell verschriftlicht, es wurde also zum einen die gesprochen-sprachlichen Anteile des Videos transkribiert und zum anderen festgehalten, welche Bilder bzw. Szenen dazu jeweils gezeigt werden. Ein Ausschnitt aus der auf diese Weise entstandenen Tabelle, die als Grundlage unserer Analysen fungiert, sei im Folgenden zu Veranschaulichungszwecken abgebildet:

\begin{tabular}{|c|c|c|}
\hline \# & AUDIO & VISUALISIERUNG \\
\hline 01 & ((langsame Musik)) & \\
\hline 02 & Das ist die BASF. & $\begin{array}{l}\text { Videoaufnahme aus der Vogelperspektive, } \\
\text { sonniger Tag, Werksübersicht am Rhein. }\end{array}$ \\
\hline 03 & $\begin{array}{l}\text { Sie wurde } 1865 \text { gegründet. Damals hieß sie } \\
\text { noch ,Badische Anilin und Soda Fabrik‘, } \\
\text { daraus wurde kurz, BASF - , The Chemical } \\
\text { Company‘. }\end{array}$ & $\begin{array}{l}\text { Unterstützung des Hörprozesses durch } \\
\text { Visualisierung durch sprachliche Zeichen } \\
\text { und Numeralien; aktuelles Logo der BASF. }\end{array}$ \\
\hline 04 & $\begin{array}{l}\text { Die BASF beschäftigt in mehr als } 80 \text { Län- } \\
\text { dern der Welt über } 110.000 \text { Mitarbeiter, } \\
\text { damit ist sie der größte Chemiekonzern der } \\
\text { Welt. }\end{array}$ & $\begin{array}{l}\text { Weltkarte (schwarz-weiß) mit Niederlas- } \\
\text { sungen (bunt), Unterstützung des Hörpro- } \\
\text { zesses durch Visualisierung der Zahlen, } \\
\text { Balkengrafik (BASF als größter Chemie- }\end{array}$ \\
\hline
\end{tabular}

\footnotetext{
${ }^{4}$ Laut A. Reins (2006: 63ff.) sind diese Elemente ein Perspektivenwechsel vom USP zum UBP (unique buying proposition), d. h. die Kommunikation fokussiert nicht die Alleinstellung des Produkts oder des Unternehmens, sondern die Kaufargumente (welche Vorteile hat das Produkt für den Kunden, welche Bedürfnisse/Wünsche hat der Kunde?) bzw. Vorzüge für den (potentiellen) Mitarbeiter (Karrierechancen, attraktive Vergütung, komfortabler Arbeitsplatz etc.).
} 


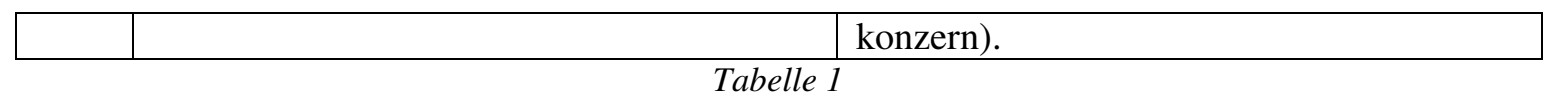

Das Video gliedert sich deutlich in zwei Teile: Während in den ersten 50 Sekunden das Unternehmen vorgestellt wird und der Zuschauer Informationen über die Gründung, die Größe, die weltweiten Niederlassungen und Produkte der BASF erhält, fungiert die zweite Hälfte, also die verbleibenden 55 Sekunden des Videos, als Bewerbungsaufruf an potenzielle Mitarbeiter (Praktikanten). In dieser Aufteilung spiegeln sich auch die oben dargelegten Ziele bzw. Aufgaben von Unternehmen, nämlich die Inszenierung einerseits und die Adressierung von Rezipienten andererseits, wider: Während im ersten Teil des Videos in erster Linie das Unternehmen BASF positiv präsentiert werden soll, steht im zweiten Teil die Ansprache des Zuschauers im Vordergrund. ${ }^{5}$ Entsprechend soll im Folgenden auch zunächst auf die kommunikativen Inszenierungsstrategien im ersten Teil des Videos eingegangen werden, um anschließend die sprachlichen Mittel der Adressierung im zweiten Teil des Videos näher zu beleuchten.

\section{Inszenierung}

Der Ausdruck ,Inszenierung' stammt aus dem Bereich des Theaters und bezeichnet dort eine Strategie, um etwas oder sich selbst in Szene zu setzen mit dem Ziel, auf diese Weise einen bestimmten Eindruck beim Rezipienten zu erzeugen (B. Husmann 2008: 30) - wobei dieser Eindruck nicht zwingenderweise der Realität entsprechen muss (vgl. etwa unsere obigen Ausführungen zum Soll- und Ich-Zustand). Solche Inszenierungstechniken finden nicht nur in der Unternehmenskommunikation, sondern in vielen weiteren Bereichen ihre Anwendung, so beispielsweise auch in der Pädagogik. In seiner Einführung in die Schulpädagogik befasst sich H. Meyer (2002) mit der Diversität von Inszenierungstechniken und nennt in diesem Rahmen exemplarisch die Strategien informieren, dramatisieren, personalisieren, vergleichen, verfremden oder parodieren. Daran angelehnt sollen im Folgenden die Inszenierungsmuster bzw. -techniken historisieren, quantifizieren, vergleichen und Herstellung des Bezugs zum Alltag der Rezipienten dargestellt werden, die, wie sich zeigen soll, im untersuchten Imagevideo der BASF eine zentrale Rolle einnehmen. Im Fokus der Analyse sollen insbesondere die sprachlichen Mittel der Inszenierung stehen.

\footnotetext{
${ }^{5}$ Jedoch sind selbstverständlich auch im ersten Teil des Videos bereits Aspekte der Adressierung zu vermerken, ebenso wie auch in der zweiten Hälfte Inszenierungsstrategien betrieben werden.
} 
Das Video beginnt mit einer Aufnahme des Firmengeländes der BASF aus der Vogelperspektive. Die erste Information, die anschließend über das Unternehmen gegeben wird, ist deren Gründungsjahr sowie der ursprüngliche Name des Unternehmens:

\begin{tabular}{|l|l|l|}
\hline 03 & $\begin{array}{l}\text { Sie wurde 1865 gegründet. Damals hieß } \\
\text { sie noch ,Badische Anilin und Soda Fab- } \\
\text { rik', daraus wurde kurz, BASF - ,The } \\
\text { Chemical Company'. }\end{array}$ & $\begin{array}{l}\text { Visualisierung der Numeralie 1865 und des } \\
\text { Firmennamens Badische Anilin und Soda } \\
\text { Fabrik sowie BASF - The Chemical Com- } \\
\text { pany, aktuelles Logo der BASF wird ein- } \\
\text { geblendet. }\end{array}$ \\
\hline
\end{tabular}

Tabelle 2

Auf diese Weise wird auf die lange Tradition des Unternehmens verwiesen, die Nennung des Gründungsjahres der BASF dient so als „Gütesiegel für ihre Beständigkeit“ (S. Burel i.Dr.): Denn durch den Hinweis auf das Gründungsjahr wird gleichzeitig auf das ,hohe Alter' des Unternehmens verwiesen und somit nicht nur dessen Erfahrung aufgezeigt, sondern insbesondere Merkmale wie „,Verlässlichkeit“ und damit ,Glaubwürdigkeit” beim Rezipienten aufgerufen“ (S. Burel i.Dr.). Die Bezugnahme auf die Geschichte des Unternehmens kann also gemeinsam mit H.-G. Gadamer (1990: 285) als Referenz auf eine „namenlos gewordene Autorität" verstanden werden. Doch nicht nur durch den (impliziten) Hinweis, dass es sich bei der BASF um ein Unternehmen handelt, das bereits seit beinahe 150 Jahren erfolgreich agiert, sondern auch durch die Nennung des ursprünglichen Namens des Unternehmen - auf dem auch der aktuelle Name BASF beruht - wird auf dessen historische Wurzeln verwiesen. Das Merkmal der Historizität wird hier also gezielt zur Inszenierung eingesetzt, um das Unternehmen glaubwürdig und vertrauenswürdig darzustellen und auf dessen Expertise zu verweisen, die wiederum aus der langjährigen Tradition des Unternehmens herrührt.

Eine weitere Inszenierungsstrategie, die in dem Imagevideo von zentraler Bedeutung ist, ist die Quantifizierung des Unternehmens, d.h. die Beschreibung des Unternehmens anhand von Numeralien: So werden in der ersten Hälfte des Videos eine Reihe von Zahlen genannt, die entsprechenden Abschnitte sind im Folgenden abgebildet:

\begin{tabular}{|l|l|l|}
\hline 04 & $\begin{array}{l}\text { Die BASF beschäftigt in mehr als 80 Län- } \\
\text { dern der Welt über 110.000 Mitarbeiter, } \\
\text { damit ist sie der größte Chemiekonzern der } \\
\text { Welt. }\end{array}$ & $\begin{array}{l}\text { Weltkarte (schwarz-weiß) mit Niederlas- } \\
\text { sungen (Bunt), Unterstützung des Hörpro- } \\
\text { zesses durch Visualisierung der Zahlen, } \\
\text { Balkengrafik (BASF als größter Chemie- } \\
\text { konzern). }\end{array}$ \\
\hline
\end{tabular}

\begin{tabular}{|l|l|lll|}
\hline 07 & Auf über 10 qm Fläche führen 115 km Stra- & Unterstützung des Hörprozesses durch \\
\hline
\end{tabular}




\begin{tabular}{|l|l|l|l|}
\hline \multicolumn{2}{|l|}{$\begin{array}{l}\text { Be, } 213 \mathrm{~km} \text { Bahngleise, 2000 km Rohrlei- } \\
\text { tungen und stehen 2000 Gebäude. }\end{array}$} & $\begin{array}{l}\text { Visualisierung der Zahlen und } \\
\text { Piktrogramme. }\end{array}$ \\
\hline 11 & $\begin{array}{l}\text { Weltweit meldet sie jährlich über 1000 neue } \\
\text { Patente an. }\end{array}$ & $\begin{array}{l}\text { Unterstützung des Hörprozesses durch } \\
\text { Visualisierung der Numeralien und Wör- } \\
\text { ter sowie durch und Abbildungen. }\end{array}$ \\
\hline
\end{tabular}

Tabelle 3

Durch die Zahlen, mit denen hier auf hohe Referenzmengen (mehr als 80 Länder, über 110.000 Mitarbeiter etc.) verwiesen wird, soll den Rezipienten die Größe des Unternehmens sowie dessen Erfolg und Expertentum vor Augen geführt werden. Gleichzeitig wird auf diese Weise beim Rezipienten das Konzept >Quantität als Legitimationsquelle< $(\mathrm{S}$. Burel i.Dr.) aufgerufen: Denn ein Unternehmen, dass sich durch eine solch hohe Anzahl an Niederlassungen, Mitarbeitern und angemeldeten Patenten auszeichnet, ist nicht nur erfolgreich und produktiv, sondern somit immer auch glaubwürdig.

Mit der Quantifizierung des Unternehmens geht auch das Inszenierungsmuster des Vergleichs einher: So wird die Größe und der Erfolg des Unternehmens nicht nur durch Zahlen gemessen, sondern auch durch einen - wenn auch impliziten - Vergleich mit anderen Unternehmen verdeutlicht. Dies geschieht hier in Form von Superlativen: So wird von der BASF als größtem Chemiekonzern der Welt bzw. als eines der innovativsten Chemieunternehmen der Welt gesprochen und die Niederlassung in Ludwigshafen als eines der größten zusammenhängenden Chemieareale auf unserem Planeten bezeichnet.

\begin{tabular}{|l|l|l|}
\hline 04 & $\begin{array}{l}\text { Die BASF beschäftigt in mehr als 80 Län- } \\
\text { dern der Welt über 110.000 Mitarbeiter, } \\
\text { damit ist sie der größte Chemiekonzern der } \\
\text { Welt. }\end{array}$ & $\begin{array}{l}\text { Weltkarte (schwarz-weiß) mit Niederlas- } \\
\text { sungen (bunt), Unterstützung des Hörpro- } \\
\text { zesses durch Visualisierung der Numera- } \\
\text { lien, Balkengrafik (BASF als größter } \\
\text { Chemiekonzern). }\end{array}$ \\
\hline 05 & $\begin{array}{l}\text { Das Werk in Ludwigshafen bildet eines der } \\
\text { größten zusammenhängenden Chemieareale } \\
\text { auf unserem Planeten: }\end{array}$ & $\begin{array}{l}\text { Videoaufnahmen des Werks aus der Vo- } \\
\text { gelperspektive. }\end{array}$ \\
\hline 10 & $\begin{array}{l}\text { Die BASF gehört zu den innovativsten } \\
\text { Chemieunternehmen der Welt. }\end{array}$ & $\begin{array}{l}\text { Mitarbeiter der BASF im Unterneh- } \\
\text { mensbereich Agriculture werden bei } \\
\text { der Arbeit gezeigt. }\end{array}$ \\
\hline
\end{tabular}

Tabelle 4

Es zeigt sich, dass hier nicht explizit Bezug auf andere Unternehmen genommen wird, von denen sich die BASF abzugrenzen versucht. Vielmehr geht es, ähnlich wie bei der zuvor beschriebenen Strategie der Quantifizierung, um die positive Darstellung des 
Unternehmens durch die Hervorhebung von dessen Größe und Qualität, weniger um die Diskreditierung von vergleichbaren Mitbewerbern. ${ }^{6}$

Eine letzte Inszenierungsstrategie, auf die eingegangen werden soll, ist die Herstellung des Bezugs zum Alltag der Rezipienten. So nennt das Unternehmen im Rahmen der Vorstellung seiner Produkte vor allem alltägliche Gegenstände bzw. Gebrauchsartikel wie Kaugummis, Jeans oder Sonnencreme und stellt auf diese Weise einen Bezug zur Lebenswelt der Rezipienten her:

\begin{tabular}{|l|l|l|}
\hline 12 & $\begin{array}{l}\text { Sie hat nicht nur den Styropor erfunden, } \\
\text { sondern auch den Kunststoff in Kaugum- } \\
\text { mis, die Farbe in Bluejeans oder das Licht- } \\
\text { schutzmittel in Sonnencreme. }\end{array}$ & $\begin{array}{l}\text { Unterstützung des Hörprozesses durch } \\
\text { Visualisierung der Numeralien und Wör- } \\
\text { ter bzw. durch Videoausschnitte von } \\
\text { jungen Menschen, die diese Produkte } \\
\text { nutzen. }\end{array}$ \\
\hline
\end{tabular}

Tabelle 5
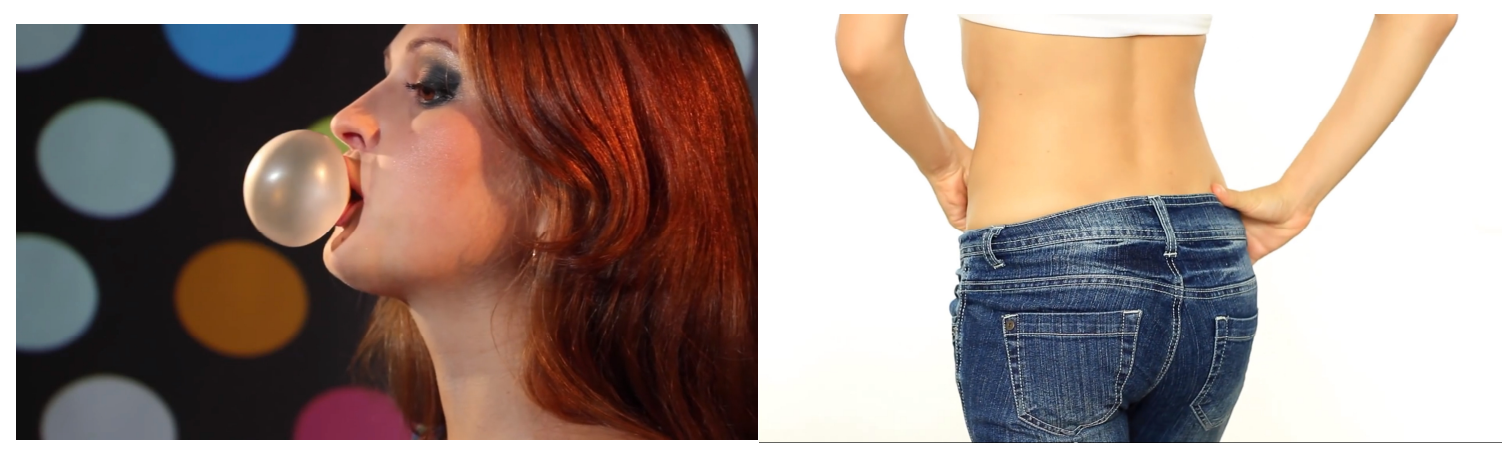

Abbildung 1: Abbildungen verschiedener Produkte, in denen Erfindungen der BASF Anwendung finden

Da auf die Dominantsetzung von Elementen aus der Lebenswelt junger Menschen jedoch im Rahmen der Analyse der Adressierungsstrategien des Imagevideos ausführlicher Bezug genommen werden soll, sei es an dieser Stelle bei diesem Hinweis belassen. Zusammenfassend kann festgehalten werden, dass in dem untersuchten Imagevideo verschiedene Inszenierungsstrategien angewendet und miteinander kombiniert werden, die als „Werkzeuge“ aus dem oben dargestellten „Werkzeugkasten“ (M. Piwinger/ H. Ebert 2001: 3) des Impression Managements zu verstehen sind, mit denen also der Eindruck, den das Unternehmen auf andere macht, gezielt gesteuert und kontrolliert werden soll (vgl. M. Piwinger/ H. Ebert 2001: 1).

\section{Adressierung}

\footnotetext{
${ }^{6}$ Diesbezüglich gilt es jedoch anzumerken, dass andere Unternehmen bzw. deren Produkte in Deutschland allerdings bereits aus rechtlichen Gründen nicht herabgesetzt werden dürfen (vgl. UWG 2010, §6 sowie ausführlicher hierzu S. Burel i.Dr.).
} 
Bevor die Analyse der Adressierung im Video besprochen werden soll, ist es zunächst wichtig, die spezifische Kommunikationssituation genauer zu bestimmen. Im ersten Teil der empirischen Analyse wurde bereits herausgestellt, wie sich das Unternehmen selbst inszeniert. Daneben scheint die Suche nach Praktikanten im Vordergrund zu stehen. Das Video kann dabei als ein Teil der Recruiting-Strategie des Unternehmens BASF identifiziert werden. Ziel der Interaktion zwischen dem Unternehmen und der angesprochenen Zielgruppe ist hier, das Handlungsmuster >Bewerbung < durch bestimmte kommunikative Mittel bei den Adressierten zu aktivieren.

Bereits im Titel ist die erste direkte Adressierung zu finden: „(...) BASF sucht Dich!“‘. Die augenfällige Du-Ansprache, die auch im Video ihre Fortsetzung findet, trägt zum einen einer jungen Zielgruppe Rechnung und schafft zum anderen Nähe in der Beziehung zwischen dem Unternehmen und den Adressierten, die auf diese Weise als selbstverständlich suggeriert wird. Eine solche Nähe zu einem Chemiekonzern ist bei einer jungen Zielgruppe normalerweise nicht zu erwarten. Das Unternehmen muss deshalb durch die sprachliche Kodierung der Ansprache den ersten Schritt auf die Adressierten zu machen, um die Kontaktaufnahme zu erleichtern. Für den weiteren Erfolg der kommunikativen Absicht ist die Perspektivübernahme von entscheidender Bedeutung und lässt sich im Video sehr gut nachverfolgen. Voraussetzung dafür ist, wie oben bereits erläutert, die Kenntnis der sprachgemeinschaftlich geltenden Konventionen der Adressierten. Entsprechend lässt sich die Sprache im Video in einen jugendsprachlichen Duktus einordnen. Sehr markant ist die Verwendung des Syntagmas „Irgendwas mit X“ (siehe Tabelle 7). Die BASF sucht einen Bewerber, der ,,irgendwas mit (...) Personal, Medien, Marketing oder Kommunikation“ studiert. Das Syntagma „,irgendwas mit X“ ist vor allem aus dem situativen Kontext der Studienwahl bekannt, wenn junge Schulabgänger nur eine vage Vorstellung davon haben, was sie studieren wollen. Im Video wird eine solche etablierte sprachliche Formel aus der Lebenswelt junger Menschen verwendet und zur Konstruktion kommunikativer Nähe instrumentalisiert. An einer späteren Stelle des Videos wird bei der Vorstellung der Voraussetzungen, die ein Praktikant mitbringen sollte, die Wichtigkeit von Karriere betont, doch in der kommunikativen Darstellung wird der Ausdruck „karrierebewusst“ gezielt vermieden: „Bei Headhunter denkst du nicht an den hier, sondern an Karriere und bei Monster denkst du nicht an den Typen, sondern an Jobbörse“.

\begin{tabular}{|l|l|l|}
\hline 18 & Bei Headhuntern & $\begin{array}{l}\text { Visualisierung des Wortes Headhunter } \\
\text { mittels sprachlicher Zeichen, } \\
\end{array}$ \\
& Einblendung eines Bildes von Boba Fett \\
\hline
\end{tabular}




\begin{tabular}{|l|l|l|}
\hline & & (Kopfgeldjäger aus dem Film Star Wars) \\
& sondern an Karriere. & Visualisierung des Wortes: Karriere. \\
\hline 19 & Bei Monster & $\begin{array}{l}\text { Visualisierung des Wortes Monster, mittels } \\
\text { sprachlicher Zeichen }\end{array}$ \\
& nicht an den Typen, & $\begin{array}{l}\text { Einblendung von Boris Karloff als Fran- } \\
\text { kenstein, }\end{array}$ \\
& Visualisierung des Wortes Jobbörse mittels \\
& sondern an Jobbörsen. & sprachlicher Zeichen. \\
\hline
\end{tabular}

Tabelle 6

Der Karriereanspruch wird mit jugendsprachlichen Konstrukten wie „den Typen“ ausgedrückt und multimedial unterstützt durch Bilder aus den Filmen Star Wars und Frankenstein (siehe Tabelle 6). Bei der Vorstellung des Unternehmens werden einige Produkte der BASF erwähnt. Dabei werden nicht etwa chemische Fachtermini verwendet, sondern alltagsnahe Gegenstände eingeblendet wie etwa Kaugummi oder Blue-Jeans (s.o.).

Es wird deutlich, dass sowohl auf sprachlicher Seite als auch auf der visuellen Bildebene Elemente aus der Lebenswelt junger Menschen dominant gesetzt werden. Es findet dabei eine Vermittlung zwischen einem globalen Chemieunternehmen, das sich sonst nur schwer in Konkreta vorstellen lässt, und den jungen Adressierten statt. Diese Vermittlung vollzieht sich durch die Kombination der oben dargestellten sprachlichen Mittel sowie der Bilder und führt zur Optimierung der Sozialdeixis und der Aktivierung des gewünschten Handlungsmusters auf Seite der Adressierten.

Die Einfühlung in die Zielgruppe zeigt sich auch in der Beschreibung der Anforderungen an einen Praktikanten: Nachdem als erstes klar gemacht wurde, aus welchem fachlichen Bereich die Bewerber kommen sollen (Personal, Medien, Marketing, Kommunikation), werden folgende Kriterien genannt: sehr gutes Englisch, Organisationsfähigkeit, Selbstständigkeit, Fähigkeit sich die Schuhe zu binden, Karriereorientiertheit (s.o.) und Netzwerken auf Onlineplattformen, wie Facebook oder Xing:

\begin{tabular}{|c|c|c|}
\hline 17 & $\begin{array}{l}\text { Du studierst irgendwas mit Schwerpunkt } \\
\text { Personal, Marketing, Medien oder Kom- } \\
\text { munikation, } \\
\text { dein Englisch ist blendend, } \\
\text { organisieren liegt dir im Blut, } \\
\text { du bist selbstständiges Arbeiten gewohnt } \\
\text { und bindest dir morgens selbst die }\end{array}$ & $\begin{array}{l}\text { Unterstützung des Hörprozesses durch Vi- } \\
\text { sualisierung der Worte Personal, Marke- } \\
\text { ting, Kommunikation, Medien, Englisch, } \\
\text { Organisieren und Selbständig mittels } \\
\text { sprachlicher Zeichen; kurze Videosequenz } \\
\text { eines Jungen im Pyjama, der sich die Schu- } \\
\text { he anzieht. }\end{array}$ \\
\hline
\end{tabular}


Auffällig ist hier, dass es kein Kriterium gibt, welches so spezifisch formuliert ist, dass eine große Zahl von potentiellen Bewerbern aus diesem Raster fällt. Der Adressiertenkreis wird bewusst vage gehalten, um eine größtmögliche Reichweite zu erlangen. Gleichzeitig wird mit dem Kriterium „Fähigkeit, sich die Schuhe zu binden“ auf komödiantische Weise ein Faktor genannt, der die Ernsthaftigkeit einer Bewerbung gezielt schmälert und eine jugendliche Lockerheit vermitteln soll. Das Unternehmen präsentiert sich auf diese Weise auf Augenhöhe mit den Adressierten.

Im Weiteren gibt das Video Auskunft darüber, welche Aufgaben auf zukünftige Praktikanten zukommen: Arbeit im European Recruiting Center, Pflege der Social Media Kanäle, Erstellung von Imagekampagnen und Optimierung von Rekrutierungskanälen:

\begin{tabular}{|l|l|ll|}
\hline 21 & $\begin{array}{l}\text { Denn hier, im European Recruiting } \\
\text { kümmerst du dich um } \\
\text { Social Media Kanäle, } \\
\text { planst Imagekampagnen } \\
\text { und hilfst bei der Optimierung von } \\
\text { Recruitierungskanälen. }\end{array}$ & $\begin{array}{l}\text { Visualisierung der Wörter European } \\
\text { Recruiting, }\end{array}$ & \\
Social Media Kanäle, & & \\
Imagekampagnen, & Recruitierungskanäle mittels sprachlicher \\
Zeichen & & \\
\hline
\end{tabular}

Tabelle 8

Anders als zuvor werden hier sprachliche Ausdrücke aus der Fachsprache (hier aus dem Personalmanagement sowie der Kommunikationswissenschaft) bemüht. Der höhere Grad an Fachlichkeit soll zeigen, dass das Unternehmen eine Expertise in bestimmten professionellen Bereichen bereitstellen kann. Das Unternehmen signalisiert auf diese Weise implizit seine Funktion als Wissensträger und kann den Adressierten vermitteln, dass es sich lohnt, genau dort ein Praktikum zu machen. Erneut wird die Perspektivübernahme deutlich, da bestimmte Erwartungen der Adressierten antizipiert und geschickt als erfüllbar dargestellt werden. Das Unternehmen suggeriert der Zielgruppe auf diese Weise eine gewisse Fürsorgeverantwortung, die S. Burel (i.Dr.) folgendermaßen beschreibt: „Zielgruppen werden zu Benefizienten, indem der Sachverhalt nicht aus Produzentensicht, sondern aus Adressiertensicht perspektiviert wird“.

Sprachlich ist nun eine leichte Spannung zwischen jugendsprachlichen Ausdrücken auf der einen Seite und fachsprachlichen Termini auf der anderen Seite zu erkennen. Diese Vermischung von Merkmalen verschiedener Varietäten ist spezifisch auf die Adressierten zugeschnitten, um einen Spagat zwischen der Herstellung kommunikativer Nähe und den professionellen Ansprüchen eines weltweit agierenden Unternehmens zu leisten. Am Ende des Videos wird mit folgender Abschlussformulierung der Bogen von den 
Adressierten als Bewerbern zu potentiellen Teammitgliedern geschlagen: „Du findest, das trifft auf dich zu? Wunderbar. Nimm dir vier bis sechs Monate Zeit und werde Teil des BASF-Teams“. Das Unternehmen stellt die Aufnahme in ein Team in Aussicht und die Hürde der Bewerbung wird dabei bewusst als niedrig dargestellt. Es entsteht der Eindruck, dass das Unternehmen genau auf den Adressierten warten würde. Dies wird noch bestärkt durch die persönliche Vorstellung durch Fotos der Praktikumsbetreuer, die sich auf die Praktikanten „freuen“. Auf dem Foto der Praktikumsbetreuerin wird ein grüner Balken eingeblendet, der diese als „Franziska, deine Praktikumsbetreuerin“ vorstellt. Die Adressierten haben so konkret vor Augen, welche Personen auf sie „warten“; die bisher als unpersönlich vorgestellte BASF bekommt auf diese Weise Gesichter und Namen.

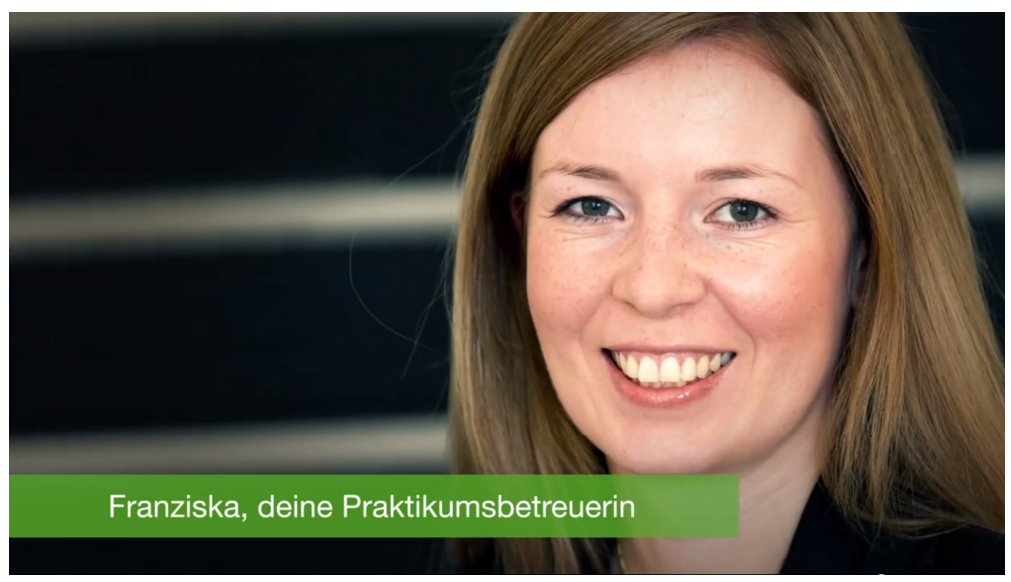

Abbildung 2: Bild der Praktikumsbetreuerin

Der Text auf dem Bild ist ganz spezifisch an einen potenziellen Bewerber gerichtet (,deine Praktikumsbetreuerin") und durch die Vorstellung nur mit Vornamen wird ein nahes persönliches Verhältnis prognostiziert. Die Sozialdeixis des Unternehmens wird auf eine persönliche Ebene gebracht und komplettiert so das Bemühen um die kommunikative Nähe.

\section{Fazit}

Es konnte gezeigt werden, dass sich im Video verschiedene multimedial-kommunikative Mittel finden lassen, die der Inszenierung des Unternehmens einerseits und der Adressierung einer bestimmten Zielgruppe andererseits dienen. Durch die Inszenierungsstrategien historisieren, quantifizieren, vergleichen und Herstellung des Bezugs zum Alltag der Rezipienten werden die Ziele des Impression Managements verfolgt, den Eindruck, den das Unternehmen auf andere macht, zu steuern. Dementsprechend wird das Unternehmen als 
erfolgreich, erfahren, kompetent und somit letztlich immer auch als glaubwürdig und verlässlich dargestellt.

Bezüglich der Adressierung fällt zuallererst die Du-Ansprache im Titel des Videos auf, die damit suggerierte Augenhöhe zwischen den Adressierten und dem Unternehmen findet im jugendlichen Sprachgebrauch und den alltagsnahen Bezugsgegenständen (Kaugummi, Bluejeans) ihre Fortsetzung. Die fachsprachlichen Anteile des Textes zeigen an, dass das Unternehmen als Wissensträger die Erwartungen der Zielgruppe erfüllen kann. Die persönliche Ebene wird schließlich in besonderer Weise durch die persönliche Vorstellung der Praktikumsbetreuer stark gemacht.

Die dargestellten Adressierungen legen zusammen mit den beschriebenen Anforderungen an Praktikanten eine primär offensichtliche Zielgruppe des Videos nahe, da sie sich auf einen ähnlichen Personenkreis beziehen. Obwohl die Anforderungen eher unspezifisch bleiben, geben sie doch einen klaren Rahmen für potenzielle Bewerber. Hier stellen sich jedoch die Fragen, ob die Zielgruppe mit der Beschreibung von Bewerbungskandidaten gleichzusetzen ist und ob das Unternehmen wirklich die Perspektive von Adressierten einnimmt oder nicht vielmehr selbst die Zielgruppen konstituiert, um implizit eigene Unternehmensinteressen zu verfolgen. Gerade in Situationen, in denen Unternehmensinteressen und Erwartungen von Zielgruppen nicht zusammenkommen, scheint eine authentische Perspektivübernahme besonders wichtig (s.o.). Eigeninteressen des Unternehmens könnten hier im Kontext der Selbstinszenierung liegen: Das Unternehmen will zeigen, dass es auf den Nachwuchs baut und somit zukunftsorientiert ist. Angesichts dieses Inszenierungsinteresses muss die Frage nach der Zielgruppenorientierung jedoch neu gestellt werden, da sich das Video mit dieser Absicht auch an Kunden sowie an weitere Stakeholder, im Sinne der Mehrfachadressierung (Mitarbeiter, Aktionäre, Journalisten, Interessierte etc.) (vgl. S. Burel i.D.) - richten könnte.

\section{Literatur}

Bergmann, R. (2011): Von der Symbiose zur effektiven Protokooperation: Möglichkeiten und Wege des Wissenstransfers zwischen Geistes- und Technikwissenschaften. Hamburg: III Internationaler Hamburger Congress zur Wirtschaftskommunikation (IHCW), 08.09.2011.

Bromme, R./ R. Jucks/ R. Rambow (2004): Experten-Laien-Kommunikation im Wissensmanagement. In: G. Reinmann/ H. Mandl, (Hrsg.), Der Mensch im Wissensmanagement: Psychologische Konzepte zum besseren Verständnis und Umgang mit Wissen. Göttingen. 176-188.

Bruhn, M. (2003): Integrierte Unternehmens- und Marktkommunikation. 3. Aufl., Stuttgart. 
Bruhn, M. (2009): Kommunikationspolitik. Systematischer Einsatz der Kommunikation für Unternehmen. (= Vahlens Handbücher der Wirtschafts- und Sozialwissenschaften) 5., aktual. Aufl., München.

Burel, S. (i.D): Der Identitätskurs der DAX-30-Unternehmen. Sprachliche Konstruktion von Selbstbildern in Repräsentationstexten. Universität Heidelberg: Dissertation.

Diaz-Bone, R. (2009): Qualitätskonvention als Diskursordnungen in Märkten. In: R. DiazBone/ G. Krell (Hrsg.), Diskurs und Ökonomie. Diskursanalytische Perspektiven auf Märkte und Organisationen. (= SpringerLink: Bücher) Wiesbaden. 267-292.

Ebert, H./ K.-P. Konerding (2008): Sprachwandel, Organisationswandel, kultureller Wandel - Aspekte eines ganzheitlichen Modells für ein Kommunikations-Controlling. In: R. Crijns/ J. Thalheim (Hrsg.), Kooperation und Effizienz in der Unternehmenskommunikation. Inner- und außerbetriebliche Kommunikationsaspekte von Corporate Identity und Interkulturalität. (= Europäische Kulturen in der Wirtschaftskommunikation 8) Wiesbaden. 67-81.

Felder, E. (2008): Forschungsnetzwerk ,Sprache und Wissen`. In: Zeitschrift für Germanistische Linguistik 36/2. 270-276.

Fix, U. (2012): Politische Identität durch Sprachkunst. In: M. Müller/ S. Kluwe (Hrsg.), Identitätsentwürfe in der Kunstkommunikation. Studien zur Praxis der sprachlichen und multimodalen Positionierung im Interaktionsraum „Kunst“. (= Sprache und Wissen 10) Berlin [u.a.]. 78-103.

Goffman, E. (1969): Wir alle spielen Theater. Die Selbstdarstellung im Alltag. (= Piper Studienausgabe) 1. Aufl., München.

Gottschalk-Mazouz, N. (2007): Was ist Wissen? Überlegungen zu einem Komplexbegriff an der Schnittstelle von Philosophie und Sozialwissenschaften. In: Sabine Ammon/ C. Heineke/ K. Selbmann [et al.] (Hrsg.): Wissen in Bewegung. Vielfalt und Hegemonie in der Wissensgesellschaft. 1. Aufl., Weilerswist. 21-40.

Gadamer, H.-G. (1990): Erhebung der Geschichtlichkeit des Verstehens zum hermeneutischen Prinzip. In: Ders.: Hermeneutik 1. Wahrheit und Methode. Grundzüge einer philosophischen Hermeneutik. 270-312

Husmann, B. (2008): Inszenierung und Unterricht. Oder man kann nicht nicht inszenieren. In: T. Klie/ S. Leonhard (Hrsg.), Performative Religionsdidaktik. Stuttgart. 26-37.

Liebert, W.-A. (2003): Wissenskonstruktion als poetisches Verfahren. In: S. Geideck/ W.A. Liebert (Hrsg.), Sinnformeln. Linguistische und soziologische Analysen von Leitbildern, Metaphern und anderen kollektiven Orientierungsmustern. (= Linguistik Impulse und Tendenzen 2) Berlin [u.a.]. 83-104.

Meyer, H. (2002): Unterrichtsmethoden. In: H. Kiper/ H. Meyer/ W. Topsch, Einführung in die Schulpädagogik. Berlin. 109-121.

Nonaka, I./ H. Takeuchi (1997): Die Organisation des Wissens. Wie japanische Unternehmen eine brachliegende Ressource nutzbar machen. Frankfurt am Main [u.a.].

Piwinger, M./ H. Ebert (2001): Impression Management - Wie aus Niemand Jemand wird. In: G. Bentele/ M. Piwinger/ G. Schönborn (Hrsg.): Kommunikationsmanagement. Strategien, Wissen, Lösungen. Neuwied; Kriftel. 1.06.

Reins, A. (2006): Corporate Language: wie Sprache über Erfolg oder Misserfolg von Marken und Unternehmen entscheidet. Mainz. 
Scherner, M. (1997): Text - Grundbegriff der Deutschdidaktik. In: M. Becker-Mrotzek (Hrsg.), Werkstattbuch Deutsch. Texte für das Studium des Faches. (= Münsteraner Einführungen: Germanistische Arbeitsbücher 1) Münster [u.a.]. 47-66.

Schmid, B.F./ B. Lyczek (2008): Die Rolle der Kommunikation in der Wertschöpfung der Unternehmung. In: M. Meckel/ B.F. Schmid (Hrsg.): Unternehmenskommunikation. Kommunikationsmanagement aus Sicht der Unternehmensführung. Wiesbaden. 3150 . 